\title{
On the Uniform Exponential Stability of Time-Varying Systems Subject to Discrete Time-Varying Delays and Nonlinear Delayed Perturbations
}

\author{
Maher Hammami, ${ }^{1}$ Mohamed Ali Hammami, ${ }^{1}$ and Manuel De la Sen ${ }^{2}$ \\ ${ }^{1}$ Faculty of Sciences of Sfax, University of Sfax, Route Soukra, BP 1171, 3000 Sfax, Tunisia \\ ${ }^{2}$ Departamento de Electricidad y Electronica Facultad de Ciencias, Universidad del Pais Vasco Leioa (Bizkaia), \\ Apartado 644, 480809 Bilbao, Spain \\ Correspondence should be addressed to Maher Hammami; hammami_maher@yahoo.fr
}

Received 9 November 2014; Accepted 19 February 2015

Academic Editor: Qingling Zhang

Copyright (C) 2015 Maher Hammami et al. This is an open access article distributed under the Creative Commons Attribution License, which permits unrestricted use, distribution, and reproduction in any medium, provided the original work is properly cited.

This paper addresses the problem of stability analysis of systems with delayed time-varying perturbations. Some sufficient conditions for a class of linear time-varying systems with nonlinear delayed perturbations are derived by using an improved Lyapunov-Krasovskii functional. The uniform global asymptotic stability of the solutions is obtained in terms of convergence toward a neighborhood of the origin.

\section{Introduction}

The problem of robust stability analysis of linear time-varying systems subject to time-varying perturbations has attracted the attention of many researchers. Explicit bounds for the structured time-varying perturbations have been derived [16] where the stability problem of linear systems subject to delayed time-varying perturbations has been studied, while only few papers [7-11] give stability conditions for linear time-varying delay systems among those [10] dealing with the exponential stability of perturbed systems. In [5], a new sufficient delay dependent exponential stability for a class of linear time-varying systems with nonlinear delayed perturbations is obtained based on a Lyapunov-Krasovskii functional. Time delay systems can include mixed neutral, discrete (or point) delays and distributed delays including Volterra-type distributed dynamics $[12,13]$. Also, delayed dynamics often appears in real-life problems like, for instance, epidemic propagation models $[14,15]$, since they affect the illness propagation via the incubation process in the studied population and the vaccination period. Delays are also useful to describe single-species population evolution models [16] and are related to certain diffusion and competition predator-prey models [17]. Conditions to preserve the asymptotic stability compared to a delay-free nominal model description have been widely studied in the literature including the case of presence of possibly delayed perturbation dynamics. See, for instance, $[1-5,7,8,11,12,18-22]$ and references therein. The main novelty of this paper relies on the fact that the proposed approach for stability analysis allows for the computation of the bounds which characterize the exponential rate of convergence of the solution towards a closed ball centered at the origin, by extending the complexity of the system by considering at the same time time-varying dynamics with time-varying time differentiable in the delays in the nominal part, by considering nonnecessarily zero lower-bounds for the delays and by considering more general conditions than just to be Lipschitz for the delayed, in general, nonlinear dynamics. Note, for instance, that the nominal part of the system has no delays in [5]; the lower-bound of the delays of the perturbations is assumed to be zero while those perturbations are assumed to be Lipschitz in the state-variables. In this paper, the nominal part is time-varying with timevarying delays, the lower-bounds of the delays can exceed zero, and the perturbations norms incorporate a time-varying upper-bound apart from the Lipschiptz type one. In [9] 
a global-null controllability is required while the asymptotic stability is not guaranteed to be of exponential type. In the same way, the asymptotic stability is not guaranteed to be exponential in [11]. Another novelty is that the delays are time-varying time-differentiable and they are not required to be known. Only lower and upper-bound of the delay functions and their time-derivatives are required for stability analysis. We will study a class of nonlinear system such that the nonlinearity is bounded by some integrable functions which are bounded, where the origin is not necessarily an equilibrium point. We deal with the practical stability of the origin (see [23]). The asymptotic stability is more important than stability, also the desired system may be unstable and yet the system may oscillate sufficiently near this state that its performance is acceptable; thus the notion of practical stability is more suitable in several situations than Lyapunov stability. In this case all state trajectories are bounded and approach a sufficiently small neighborhood of the origin. One also desires that the state approaches the origin (or some sufficiently small neighborhood of it) in a sufficiently fast manner. This notion of practical stability was introduced by [24] for nonlinear time-varying systems and studied for differential equations with delays by [25] (see also the references therein). Moreover, the authors in $[26,27]$ constructed stabilizing controllers to obtain global convergence of solutions toward a small ball for some classes of uncertain control systems. In this paper some sufficient conditions are given to obtain the exponential uniform stability of the solutions toward a neighborhood of the origin based on a suitable LyapunovKrasovskii functional. Two illustrative examples are given to demonstrate the validity of the main result, where we establish a table of comparison with other results.

\section{Preliminaries}

We start by introducing some notations and definitions that will be employed throughout the paper.

$\mathfrak{R}^{+}$denotes the set of all nonnegative real numbers; $\mathfrak{R}^{n}$ denotes the $n$-dimensional Euclidean space; $\|x\|$ denotes the Euclidean vector norm of $x \in \mathfrak{R}^{n} ; x^{T} y$ denotes the scalar product of two vectors $x, y$;

$\mathfrak{R}^{n \times r}$ denotes the space of all $(n \times r)$-matrices;

$A^{T}$ denotes the transpose of the matrix $A ; A$ is symmetric if $A=A^{T}$;

$I$ denotes the identity matrix;

$\lambda(A)$ denotes the set of eigenvalues of $A ; \lambda_{\max }(A)=$ $\max \{\mathfrak{R} e(\lambda): \lambda \in \lambda(A)\}$;

$\mu(A(t))$ denotes the matrix measure of the matrix $A$ defined by

$$
\mu(A(t))=\frac{1}{2} \lambda_{\max }\left(A(t)+A^{T}(t)\right)
$$

$L_{2}\left(\left[-\tau_{d}, 0\right], \Re^{n}\right)$ denotes the Hilbert space of all $L_{2^{-}}$ integrable and $\mathfrak{R}^{n}$-valued functions on $[0, t]$;
$C\left(\left[-\tau_{d}, 0\right], \mathfrak{R}^{n}\right)$ denotes the Banach space of all $\mathfrak{R}^{n}-$ valued continuous functions mapping $\left[-\tau_{d}, 0\right]$ into $\mathfrak{R}^{n}$ with $\tau_{d}>0$ :

$$
\begin{gathered}
x_{t}:=\left\{x(t+s), s \in\left[-\tau_{d}, 0\right]\right\}, \\
\left\|x_{t}\right\|=\sup _{s \in\left[-\tau_{d}, 0\right]}\|x(t+s)\| .
\end{gathered}
$$

$B(0, r)=\left\{x \in \mathfrak{R}^{n} /\|x\| \leq r\right\}$, with $r>0$, denotes the closed ball of center 0 and radius $r$.

Consider a linear time-varying system with nonlinear delayed perturbations of the following form:

$$
\begin{gathered}
\dot{x}(t)=A(t) x(t)+f_{0}(t, x(t))+\sum_{i=1}^{m} A_{i}(t) x\left(t-\tau_{i}(t)\right) \\
+\sum_{i=1}^{m} f_{i}\left(t, x\left(t-\tau_{i}(t)\right)\right), \quad t \geq 0, \\
x(t)=\phi(t), \quad t \in\left[-\tau_{d}, 0\right]
\end{gathered}
$$

with $\tau_{d}=\max _{1 \leq i \leq m}\left(\tau_{i H}\right)$, where $x(t) \in \mathfrak{R}^{n}$ is the vector, where $A(t), A_{i}(t) \in \mathfrak{R}^{(n, n)}, i=1, \ldots, m$ are matrices functions continuous and bounded in $t \geq 0, \phi(t) \in C\left(\left[-\tau_{d}, 0\right], \Re^{n}\right)$ is the function of initial conditions with the norm $\|\phi\|=$ $\sup _{s \in\left[-\tau_{d}, 0\right]}\|\phi(s)\| ; \tau_{i}(t)(i=1, \ldots, m)$ is a given timedifferentiable time-varying delay function satisfying

$$
\begin{gathered}
\tau_{i L}<\tau_{i}(t) \leq \tau_{i H}, \\
\dot{\tau}_{i}(t) \leq \mu<1, \quad(i=1, \ldots, m), \forall t \geq 0,
\end{gathered}
$$

where $\tau_{i L}, \tau_{i H}\left(0 \leq \tau_{i L}<\tau_{i H}\right), \tau_{i H L}=\tau_{i H}-\tau_{i L}$.

As a matter of fact, the case that the delay derivative is larger than or equal to 1 is universal. For example, in network control systems, the delay $\tau_{i}(t)$ denotes $t-j_{k}$, where $j_{k}(k=$ $1,2, \ldots)$ are the sampling instants. Thus, this kind of delay satisfies $\dot{\tau}_{i}(t)=1$ almost for all $t \geq 0$ (see [12]). For the case of $\mu \geq 1$, if choosing a positive scalar $0<\nu<\mu^{-1}$, then it follows that

$$
\left(\nu \tau_{i}(t)\right)^{\prime}=\nu \dot{\tau}_{i}(t) \leq \nu \mu<1
$$

and the nonlinear perturbation $f_{i}(\cdot, \cdot)(i=0, \ldots, m)$ satisfies

$$
\left\|f_{i}(t, y)\right\| \leq \delta_{i 1}\|y\|+\delta_{i 2}(t), \quad \forall t \geq 0, \forall y \in \mathfrak{R}^{n},
$$

where $\delta_{i 1}>0$ and $\delta_{i 2}(\cdot)$ are nonnegative continuous bounded functions for $i=0,1, \ldots, m$.

Definition 1. The system (3) is said to be globally uniformly exponentially practically stable toward a ball $B(0, r)$ of radius $r$ which is a neighborhood of the origin, if there exist positive numbers $\alpha, \gamma$, and $r$, such that every solution $x(t, \phi)$ of the system satisfies

$$
\|x(t, \phi)\| \leq \gamma e^{-\alpha\left(t-t_{0}\right)}\|\phi\|+r, \quad \forall t \geq t_{0} \geq 0 .
$$

The following technical proposition is needed for the proof of the main result. 
Proposition 2. Let $Q, S$ be symmetric matrices of appropriate dimensions and $S>0$. Then

$$
2 x^{T} Q y-y^{T} S y \leq x^{T} Q S^{-1} Q^{T} x, \quad \forall(x, y) \in \mathfrak{R}^{n} \times \mathfrak{R}^{n} .
$$

\section{Main Result}

Theorem 3. Suppose that there exist some positive constants $\alpha$, $\beta, \nu, \kappa_{1}, \kappa_{2_{1 i}}, \kappa_{2_{2 i}}, \kappa_{2_{3 i}}, \kappa_{2_{4 i}}, \kappa_{3_{1 i}}, \kappa_{3_{2 i}}$, and a symmetric bounded positive semidefinite differentiable matrix function $P(t)$ for all $t \geq 0$ satisfying the following Lyapunov differential matrix equation (see [18]):

$$
\begin{aligned}
\dot{P}(t) & +A^{T}(t) P(t)+P(t) A(t)+2 \alpha P(t) \\
& +\sum_{i=1}^{m} \frac{1}{q_{i}} P_{\beta}(t) A_{i}(t) \cdot A_{i}^{T}(t) P_{\beta}(t)+\epsilon I=0
\end{aligned}
$$

with

$$
\begin{gathered}
\epsilon=2(p+\beta) \delta_{01}+2 \alpha \beta+\sum_{i=1}^{m} \kappa_{2_{i}}+\sum_{i=1}^{m} \kappa_{3_{i}} e^{2 \alpha \tau_{i H}}+\kappa_{1}, \\
\epsilon>0, \\
\kappa_{2_{i}}=\kappa_{2_{1 i}}+\kappa_{2_{2 i}}+\kappa_{2_{3 i}}+\kappa_{2_{4 i}}, \\
\kappa_{3_{i}}=\kappa_{3_{1 i}} \tau_{i H}^{2}+\kappa_{3_{2 i}} \tau_{i H L}^{2}, \\
P_{\beta}(t)=P(t)+\beta I, \\
p=\sup _{t \in \Re^{+}}\|P(t)\|,
\end{gathered}
$$

where

$$
\begin{gathered}
\eta=\kappa_{1}-2 \beta \bar{\mu}(A) \\
-\sum_{i=1}^{m} \frac{(p+\beta)^{2} \delta_{1}^{2}}{\kappa_{2_{3 i}} e^{-2 \alpha \tau_{i H}}(1-\mu)-q_{i}}>0, \\
0<q_{i}<\kappa_{2_{3 i}} e^{-2 \alpha \tau_{i H}}(1-\mu), \quad i=1, \ldots, m, \\
0<\mu<v^{-1},
\end{gathered}
$$

with $\bar{\mu}(A)=\sup _{t \in \mathfrak{R}^{+}} \mu(A(t))$ and $\delta_{1}=\max _{1 \leq i \leq m} \delta_{i 1}$.

$$
\begin{aligned}
& \text { If } \\
\delta_{1}< & \frac{1}{(p+\beta)} \\
& \cdot\left(\sum_{i=1}^{m}\left(\kappa_{2_{3 i}} e^{-2 \alpha \tau_{i H}}(1-\mu)-q_{i}\right)\left(\kappa_{1}-2 \beta \bar{\mu}(A)\right)\right)^{1 / 2}
\end{aligned}
$$

with $\kappa_{1}-2 \beta \bar{\mu}(A)>0$ and $\delta_{2}(t)=\sum_{i=0}^{m} \delta_{i 2}(t)$, then the system (3) is globally uniformly exponentially practically stable toward a certain ball $B(0, r)$.
Moreover, the solution $x(t, \phi)$ satisfies an estimation as in (7), with size

$$
\begin{gathered}
\gamma=\left(\left(p+\beta+\sum_{i=1}^{m}\left(\kappa_{2_{1 i}} \tau_{i L}+\left(\kappa_{2_{2 i}}+\kappa_{2_{3 i}}+\kappa_{2_{4 i}} \nu\right) \tau_{i H}\right.\right.\right. \\
\left.\left.\quad+\kappa_{3_{1 i}} c_{i H} \tau_{i H}^{3}+\kappa_{3_{2 i}} \tau_{i H L} d_{i H L}\right)\right) \\
\left.\cdot(\beta)^{-1}\right)^{1 / 2}
\end{gathered}
$$

with $d_{i H L}=\left(c_{i H} \tau_{i H}^{2}-c_{i L} \tau_{i L}^{2}\right), c_{i H}=\left(2 \alpha \tau_{i H} e^{2 \alpha \tau_{i H}}+e^{-2 \alpha \tau_{i H}}-\right.$ $1) /\left(2 \alpha \tau_{i H}\right)^{2}, c_{i L}=\left(2 \alpha \tau_{i L} e^{2 \alpha \tau_{i H}}+e^{-2 \alpha \tau_{i L}}-1\right) /\left(2 \alpha \tau_{i L}\right)^{2}$, and

$$
r=r_{1}=\sqrt{\frac{M_{1}}{2 \alpha \beta}}
$$

if $\delta_{2}$ is bounded by a scalar positive $M$ for all $t \geq 0$, with

$$
M_{1}=\frac{(p+\beta)^{2} M^{2}}{\eta}
$$

(ii)

$$
r=r_{2}=\sqrt{\frac{\widetilde{I}^{1 / 2}}{2 \beta \sqrt{\alpha}}}
$$

$$
\text { if } \int_{0}^{+\infty} \delta_{2}^{4}(s) d s<+\infty \text {, with }
$$

$$
\widetilde{I}:=\int_{0}^{+\infty} \widetilde{r}^{2}(s) d s, \quad \tilde{r}(t)=\frac{(p+\beta)^{2}}{\eta} \delta_{2}^{2}(t) .
$$

Proof. Consider the following Lyapunov-Karovskii functional:

$$
V\left(t, x_{t}\right)=V_{1}(\cdot)+V_{2}(\cdot)+V_{3}(\cdot),
$$


where

$$
\begin{aligned}
& V_{1}\left(t, x_{t}\right)=x^{T} P(t) x(t)+\beta\|x(t)\|^{2}, \\
& V_{2}\left(t, x_{t}\right)=\sum_{i=1}^{m} \kappa_{2_{1 i}} \int_{t-\tau_{i L}}^{t} e^{2 \alpha(s-t)}\|x(s)\|^{2} d s \\
& +\sum_{i=1}^{m} \kappa_{2 i} \int_{t-\tau_{i H}}^{t} e^{2 \alpha(s-t)}\|x(s)\|^{2} d s \\
& +\sum_{i=1}^{m} \kappa_{2_{3 i}} \int_{t-\tau_{i}(t)}^{t} e^{2 \alpha(s-t)}\|x(s)\|^{2} d s \\
& +\sum_{i=1}^{m} \kappa_{2_{4 i}} \int_{t-\gamma \tau_{i}(t)}^{t} e^{2 \alpha(s-t)}\|x(s)\|^{2} d s, \\
& V_{3}\left(t, x_{t}\right) \\
& =\sum_{i=1}^{m} \kappa_{3_{1 i}} \tau_{i H} \int_{-\tau_{i H}}^{0} \int_{t+t_{1}-\tau_{i}\left(t+t_{1}\right)}^{t} e^{2 \alpha\left(s+\tau_{i H}-t\right)}\|x(s)\|^{2} d s d t_{1} \\
& +\sum_{i=1}^{m} \kappa_{3_{2 i}} \tau_{i H L} \int_{-\tau_{i H}}^{-\tau_{i L}} \int_{t+t_{1}-\tau_{i}\left(t+t_{1}\right)}^{t} e^{2 \alpha\left(s+\tau_{i H}-t\right)}\|x(s)\|^{2} d s d t_{1},
\end{aligned}
$$

with $\alpha>0$.

Let us consider the time derivative of $V_{1}\left(t, x_{t}\right)$,

$$
\begin{aligned}
\dot{V}_{1}\left(t, x_{t}\right)= & x^{T}(t) \dot{P}(t) x(t)+\dot{x}^{T}(t) P_{\beta}(t) x(t) \\
& +x^{T}(t) P_{\beta}(t) \dot{x}(t)
\end{aligned}
$$

with $P_{\beta}(t)=P(t)+\beta I$.

Thus,

$$
\begin{aligned}
\dot{V}_{1}\left(t, x_{t}\right)= & x^{T}(t)\left(\dot{P}(t)+A^{T}(t) P_{\beta}(t)+P_{\beta}(t) A(t)\right) x(t) \\
& +2 P_{\beta}(t)\left(f_{0}(t, x(t))+\sum_{i=1}^{m} f_{i}\left(t, x\left(t-\tau_{i}\right)\right)\right) x(t) \\
& +2 \sum_{i=1}^{m} x^{T}(t) P_{\beta}(t) A_{i}(t) x\left(t-\tau_{i}(t)\right) .
\end{aligned}
$$

From Proposition 2, we have

$$
\begin{gathered}
2 \sum_{i=1}^{m} x^{T}(t) P_{\beta}(t) A_{i}(t) x\left(t-\tau_{i}(t)\right) \\
\leq \sum_{i=1}^{m}\left(\frac{1}{q_{i}} x^{T}(t) P_{\beta}(t) A_{i}(t) \cdot A_{i}^{T}(t) P_{\beta}(t) x(t)\right. \\
\left.+q_{i}\left\|x\left(t-\tau_{i}(t)\right)\right\|^{2}\right)
\end{gathered}
$$

This implies that

$$
\begin{aligned}
\dot{V}_{1}\left(t, x_{t}\right) & \\
\leq & x^{T}(t)\left(\dot{P}(t)+A^{T}(t) P_{\beta}(t)+P_{\beta}(t) A(t)\right) x(t) \\
& +2\left(\sup _{t \in \mathcal{R}^{+}}\|P(t)\|+\beta\right) \\
& \cdot\left(f_{0}(t, x(t))+\sum_{i=1}^{m}\left\|f_{i}\left(t, x\left(t-\tau_{i}\right)\right)\right\|\right) \cdot\|x(t)\| \\
& +\sum_{i=1}^{m} \frac{1}{q_{i}} x^{T}(t) P_{\beta}(t) A_{i}(t) \cdot A_{i}^{T}(t) P_{\beta}(t) x(t) \\
& +\sum_{i=1}^{m} q_{i}\left\|x\left(t-\tau_{i}(t)\right)\right\|^{2} .
\end{aligned}
$$

It follows that

$$
\dot{V}_{1}\left(t, x_{t}\right) \leq-2 \alpha V_{1}\left(t, x_{t}\right)+x^{T}(t)
$$

$$
\cdot\left(\dot{P}(t)+A^{T}(t) P_{\beta}(t)+P_{\beta}(t) A(t)\right.
$$$$
+\sum_{i=1}^{m} \frac{1}{q_{i}} P_{\beta}(t) A_{i}(t) \cdot A_{i}^{T}(t) P_{\beta}(t)
$$$$
\left.+2 \alpha P(t)+2\left((p+\beta) \delta_{01}+\alpha \beta\right) I\right) x(t)
$$$$
+2(p+\beta) \delta_{2}(t)\|x(t)\|+2(p+\beta)
$$$$
\cdot \sum_{i=1}^{m} \delta_{1}(t)\left\|x\left(t-\tau_{i}(t)\right)\right\| \times\|x(t)\|
$$

$$
+\sum_{i=1}^{m} q_{i}\left\|x\left(t-\tau_{i}(t)\right)\right\|^{2}
$$

with

$$
p=\sup _{t \in \mathfrak{R}^{+}}\|P(t)\|,
$$$$
\delta_{1}=\max _{1 \leq i \leq m} \delta_{i 1}, \quad \delta_{2}(t)=\sum_{i=0}^{m} \delta_{i 2}(t) .
$$ 
Next, the time derivative of $V_{2}\left(t, x_{t}\right)$ is given by

$$
\begin{aligned}
\dot{V}_{2}\left(t, x_{t}\right) \leq & -2 \alpha V_{2}\left(t, x_{t}\right)+\sum_{i=1}^{m} \kappa_{2_{i}}\|x(t)\|^{2} \\
& -\sum_{i=1}^{m} \kappa_{2_{1 i}} e^{-2 \alpha \tau_{i L}}\left\|x\left(t-\tau_{i L}\right)\right\|^{2} \\
& -\sum_{i=1}^{m} \kappa_{2_{2 i}} e^{-2 \alpha \tau_{i H}}\left\|x\left(t-\tau_{i H}\right)\right\|^{2} \\
& -\sum_{i=1}^{m} \kappa_{2_{3 i}} e^{-2 \alpha \tau_{i H}}(1-\mu)\left\|x\left(t-\tau_{i}(t)\right)\right\|^{2} \\
& -\sum_{i=1}^{m} \kappa_{2_{4 i}} e^{-2 \alpha v \tau_{i H}}(1-v \mu)\left\|x\left(t-v \tau_{i}(t)\right)\right\|^{2},
\end{aligned}
$$

with $\kappa_{2_{i}}=\kappa_{2_{1 i}}+\kappa_{2_{2 i}}+\kappa_{2_{3 i}}+\kappa_{2_{4 i}}$.

The time derivative of $V_{3}\left(t, x_{t}\right)$ is given by

$$
\begin{aligned}
& \dot{V}_{3}\left(t, x_{t}\right) \\
& =-2 \alpha V_{3}\left(t, x_{t}\right) \\
& +\sum_{i=1}^{m} \kappa_{3_{1 i}} \tau_{i H}\left(\tau_{i H} e^{2 \alpha \tau_{i H}}\|x(t)\|^{2}\right. \\
& +\int_{-\tau_{i H}}^{0}\left(-\dot{u}(t+s) e^{2 \alpha\left(s-\tau_{i}(t+s)+\tau_{i H}\right)}\right. \\
& +\sum_{i=1}^{m} \kappa_{3_{2 i}} \tau_{i H L}\left(\tau_{i H L} e^{2 \alpha \tau_{i H}}\|x(t)\|^{2}\right. \\
& +\int_{-\tau_{i H}}^{-\tau_{i L}}\left(-\dot{u}(t+s) e^{2 \alpha\left(s-\tau_{i}(t+s)+\tau_{i H}\right)}\right. \\
& \left.\left.\cdot\left\|x\left(t+s-\tau_{i}(t+s)\right)\right\|^{2}\right) d s\right) .
\end{aligned}
$$

By using the following differentiation rule (see [5]), one obtains

$$
\begin{aligned}
& \frac{d}{d t}\left(\int_{-\tau_{i}}^{0} \int_{u\left(t+t_{1}\right)}^{t} \psi(s) d s d t_{1}\right) \\
& \quad=\tau_{i} \psi(t)-\int_{-\tau_{i}}^{0} \dot{u}(t+s) \psi(u(t+s)) d s,
\end{aligned}
$$

with

$$
\begin{gathered}
u(t+s)=t+s-\tau_{i}(t+s), \\
\dot{u}(t+s)=1-\dot{\tau}_{i}(t+s) .
\end{gathered}
$$

It follows that

$$
-\dot{u}(t+s) \leq \mu-1 .
$$

So,

$$
\begin{aligned}
\dot{V}_{3}\left(t, x_{t}\right) \leq & -2 \alpha V_{3}\left(t, x_{t}\right) \\
& +\left(\sum_{i=1}^{m} \kappa_{3_{1 i}} \tau_{i H}^{2}+\sum_{i=1}^{m} \kappa_{3_{2 i}} \tau_{i H L}^{2}\right) e^{2 \alpha \tau_{i H}}\|x(t)\|^{2} \\
& -\sum_{i=1}^{m} \kappa_{3_{1 i}} \tau_{i H} e^{-2 \alpha \tau_{i H}}(1-\mu) \\
& \cdot \int_{-\tau_{i H}}^{0}\left\|x\left(t+s-\tau_{i}(t+s)\right)\right\|^{2} d s \\
& -\sum_{i=1}^{m} \kappa_{3_{2 i}} \tau_{i H L} e^{-2 \alpha \tau_{i H}}(1-\mu) \\
& \cdot \int_{-\tau_{i H}}^{-\tau_{i L}}\left\|x\left(t+s-\tau_{i}(t+s)\right)\right\|^{2} d s .
\end{aligned}
$$

Since the last integral term is nonnegative, we obtain the following estimation on $\dot{V}_{3}\left(t, x_{t}\right)$ :

$$
\dot{V}_{3}\left(t, x_{t}\right) \leq-2 \alpha V_{3}\left(t, x_{t}\right)+\sum_{i=1}^{m} \kappa_{3_{i}} e^{2 \alpha \tau_{i H}}\|x(t)\|^{2},
$$

with $\kappa_{3_{i}}=\kappa_{3_{1 i}} \tau_{i H}^{2}+\kappa_{3_{2 i}} \tau_{i H L}^{2}$.

Therefore, from (24)-(26)-(32), it follows that

$$
\dot{V}\left(t, x_{t}\right) \leq-2 \alpha V\left(t, x_{t}\right)+V_{0}\left(t, x_{t}\right) \text {, }
$$

where

$$
\begin{aligned}
& V_{0}\left(t, x_{t}\right) \\
& =x^{T}(t)\left(\dot{P}(t)+A^{T}(t) P_{\beta}(t)+P_{\beta}(t) A(t)\right. \\
& +\sum_{i=1}^{m} \frac{1}{q_{i}} P_{\beta}(t) A_{i}(t) \cdot A_{i}^{T}(t) P_{\beta}(t) \\
& \left.+2 \alpha P(t)+2\left((p+\beta) \delta_{01}+\alpha \beta\right) I\right) x(t) \\
& +2(p+\beta) \delta_{2}(t)\|x(t)\|+2(p+\beta) \delta_{1} \\
& \quad \sum_{i=1}^{m}\left\|x\left(t-\tau_{i}(t)\right)\right\| \times\|x(t)\|
\end{aligned}
$$$$
+\sum_{i=1}^{m} q_{i}\left\|x\left(t-\tau_{i}(t)\right)\right\|^{2}+\sum_{i=1}^{m} \kappa_{2_{i}}\|x(t)\|^{2}
$$ 


$$
\begin{aligned}
& -\sum_{i=1}^{m} \kappa_{2_{1 i}} e^{-2 \alpha \tau_{i L}}\left\|x\left(t-\tau_{i L}\right)\right\|^{2} \\
& -\sum_{i=1}^{m} \kappa_{2_{2 i}} e^{-2 \alpha \tau_{i H}}\left\|x\left(t-\tau_{i H}\right)\right\|^{2} \\
& -\sum_{i=1}^{m} \kappa_{2_{3 i}} e^{-2 \alpha \tau_{i H}}(1-\mu)\left\|x\left(t-\tau_{i}(t)\right)\right\|^{2} \\
& -\sum_{i=1}^{m} \kappa_{2_{4 i}} e^{-2 \alpha v \tau_{i H}}(1-v \mu)\left\|x\left(t-v \tau_{i}(t)\right)\right\|^{2} \\
& +\sum_{i=1}^{m} \kappa_{3_{i}} e^{2 \alpha \tau_{i H}}\|x(t)\|^{2} .
\end{aligned}
$$

It follows that

$$
\begin{aligned}
V_{0}\left(t, x_{t}\right) & \\
\leq & x^{T}(t) \\
& \cdot\left(\dot{P}(t)+A^{T}(t) P(t)+P(t) A(t)+2 \alpha P(t)\right. \\
& +\sum_{i=1}^{m} \frac{1}{q_{i}} P_{\beta}(t) A_{i}(t) \cdot A_{i}^{T}(t) P_{\beta}(t) \\
& +\left(2(p+\beta) \delta_{01}+2 \alpha \beta+\sum_{i=1}^{m} \kappa_{2_{i}}\right. \\
& +\beta x^{T}(t)\left(A^{T}(t)+A(t)\right) x(t) \\
& +2(p+\beta) \delta_{2}(t)\|x(t)\|+2(p+\beta) \delta_{1} \\
& \left.+\sum_{i=1}^{m} \kappa_{2_{2 i}} e^{-2 \alpha \tau_{i H}}\left\|x\left(t-\tau_{3_{i}} e^{2 \alpha \tau_{i H}}\right)\right\|^{2} I\right) x(t) \\
& +\sum_{i=1}^{m}\left\|x\left(t-\tau_{i}(t)\right)\right\| \times\|x(t)\| \\
+ & \sum_{i=1}^{m} q_{i}\left\|x\left(t-\tau_{i}(t)\right)\right\|^{2} \\
& \\
& \\
& \\
& \\
& \\
&
\end{aligned}
$$

$$
\begin{aligned}
& -\sum_{i=1}^{m} \kappa_{2_{3 i}} e^{-2 \alpha \tau_{i H}}(1-\mu)\left\|x\left(t-\tau_{i}(t)\right)\right\|^{2} \\
& -\sum_{i=1}^{m} \kappa_{2_{4 i}} e^{-2 \alpha v \tau_{i H}}(1-\nu \mu)\left\|x\left(t-v \tau_{i}(t)\right)\right\|^{2} .
\end{aligned}
$$

Now, using Proposition 2, we have

$$
\begin{aligned}
\sum_{i=1}^{m} & \left(2(p+\beta) \delta_{1}\left\|x\left(t-\tau_{i}(t)\right)\right\| \cdot\|x(t)\|\right. \\
& \left.-\left(\kappa_{2_{3 i}} e^{-2 \alpha \tau_{i H}}(1-\mu)-q_{i}\right)\left\|x\left(t-\tau_{i}(t)\right)\right\|^{2}\right) \\
\leq & \sum_{i=1}^{m} \frac{(p+\beta)^{2} \delta_{1}^{2}}{\kappa_{2_{3 i}} e^{-2 \alpha \tau_{i H}}(1-\mu)-q_{i}}\|x(t)\|^{2},
\end{aligned}
$$

with

$$
0<q_{i}<\kappa_{23 i} e^{-2 \alpha \tau_{i H}}(1-\mu), \quad i=1, \ldots, m .
$$

Hence, the above expression, in conjunction with (35), yields

$$
\begin{aligned}
V_{0}\left(t, x_{t}\right) \leq & x^{T}(t) \\
& \cdot\left(\dot{P}(t)+A^{T}(t) P(t)+P(t) A(t)+2 \alpha P(t)\right. \\
& +\sum_{i=1}^{m} \frac{1}{q_{i}} P_{\beta}(t) A_{i}(t) \cdot A_{i}^{T}(t) P_{\beta}(t) \\
& +\left(2(p+\beta) \delta_{01}+2 \alpha \beta\right. \\
& -\kappa_{1} x^{T}(t) x(t)+\beta x^{T}(t)\left(A^{T}(t)+A(t)\right) x(t) \\
& +\sum_{i=1}^{m} \frac{(p+\beta)^{2} \delta_{1}^{2}}{\kappa_{2_{3 i}} e^{-2 \alpha \tau_{i H}}(1-\mu)-q_{i}}\|x(t)\|^{2} \\
& +2(p+\beta) \delta_{2}(t) \cdot\|x(t)\| \\
& \left.-\sum_{i=1}^{m} \kappa_{2_{1 i}} e^{-2 \alpha \tau_{i L}} \| x\left(t-\tau_{2_{i}}+\sum_{i=1}^{m} \kappa_{3_{i}} e^{2 \alpha \tau_{i H}}+\kappa_{1}\right) I\right) x(t) \\
& -\sum_{i=1}^{m} \kappa_{2_{2 i}} e^{-2 \alpha \tau_{i H}}\left\|x\left(t-\tau_{i H}\right)\right\|^{2} \\
& -\sum_{i=1}^{m} \kappa_{2_{4 i}} e^{-2 \alpha v \tau_{i H}}(1-v \mu)\left\|x\left(t-v \tau_{i}(t)\right)\right\|^{2} .
\end{aligned}
$$

Since $P(t)$ is a solution of $(9)$ with

$$
\epsilon=2(p+\beta) \delta_{01}+2 \alpha \beta+\sum_{i=1}^{m} \kappa_{2_{i}}+\sum_{i=1}^{m} \kappa_{3_{i}} e^{2 \alpha \tau_{i H}}+\kappa_{1},
$$


then

$$
\begin{aligned}
V_{0}( & \left.t, x_{t}\right) \\
\leq & -\kappa_{1} x^{T}(t) x(t)+\beta x^{T}(t)\left(A^{T}(t)+A(t)\right) x(t) \\
& +\sum_{i=1}^{m} \frac{(p+\beta)^{2} \delta_{1}^{2}}{\kappa_{23 i} e^{-2 \alpha \tau_{i H}}(1-\mu)-q_{i}}\|x(t)\|^{2} \\
& +2(p+\beta) \delta_{2}(t) \cdot\|x(t)\| \\
& -\sum_{i=1}^{m} \kappa_{2_{1 i}} e^{-2 \alpha \tau_{i L}}\left\|x\left(t-\tau_{i L}\right)\right\|^{2} \\
& -\sum_{i=1}^{m} \kappa_{2_{2 i}} e^{-2 \alpha \tau_{i H}}\left\|x\left(t-\tau_{i H}\right)\right\|^{2} \\
& -\sum_{i=1}^{m} \kappa_{2_{4 i}} e^{-2 \alpha v \tau_{i H}}(1-v \mu)\left\|x\left(t-v \tau_{i}(t)\right)\right\|^{2} .
\end{aligned}
$$

Note that we have

$$
\begin{aligned}
x^{T} & (t)\left(A^{T}(t)+A(t)\right) x(t) \\
& \leq \lambda_{\max }\left(A(t)+A^{T}(t)\right)\|x(t)\|^{2} \\
& \leq 2 \mu(A(t))\|x(t)\|^{2} \\
& \leq 2 \bar{\mu}(A)\|x(t)\|^{2},
\end{aligned}
$$

with $\bar{\mu}(A)=\sup _{t \in \Re^{+}} \mu(A(t))$.

Then, it follows that

$$
\begin{aligned}
V_{0}\left(t, x_{t}\right) & \\
\leq & -\left(\kappa_{1}-2 \beta \bar{\mu}(A)\right. \\
& \left.\quad-\sum_{i=1}^{m} \frac{(p+\beta)^{2} \delta_{1}^{2}}{\kappa_{23 i} e^{-2 \alpha \tau_{i H}}(1-\mu)-q_{i}}\right)\|x(t)\|^{2} \\
& +2(p+\beta) \delta_{2}(t) \cdot\|x(t)\| \\
& -\sum_{i=1}^{m} \kappa_{2_{1 i}} e^{-2 \alpha \tau_{i L}}\left\|x\left(t-\tau_{i L}\right)\right\|^{2} \\
& -\sum_{i=1}^{m} \kappa_{2_{2 i}} e^{-2 \alpha \tau_{i H}}\left\|x\left(t-\tau_{i H}\right)\right\|^{2} \\
& -\sum_{i=1}^{m} \kappa_{2_{4 i}} e^{-2 \alpha v \tau_{i H}}(1-v \mu)\left\|x\left(t-v \tau_{i}(t)\right)\right\|^{2} .
\end{aligned}
$$

Let

$$
\eta=\kappa_{1}-2 \beta \bar{\mu}(A)-\sum_{i=1}^{m} \frac{(p+\beta)^{2} \delta_{1}^{2}}{\kappa_{2_{3 i}} e^{-2 \alpha \tau_{i H}}(1-\mu)-q_{i}} .
$$

One has

$$
\begin{aligned}
& \eta>0 \\
& \Longrightarrow \delta_{1}<\frac{\sqrt{\sum_{i=1}^{m}\left(\kappa_{2_{3 i}} e^{-2 \alpha \tau_{i H}}(1-\mu)-q_{i}\right)\left(\kappa_{1}-2 \beta \bar{\mu}(A)\right)}}{(p+\beta)}, \\
& \kappa_{1}-2 \beta \bar{\mu}(A)>0 .
\end{aligned}
$$

Then,

$$
\begin{aligned}
V_{0}\left(t, x_{t}\right) \leq & -\eta(\|x(t)\|-\xi(t))^{2}+\widetilde{r}(t) \\
& -\sum_{i=1}^{m} \kappa_{2_{1 i}} e^{-2 \alpha \tau_{i L}}\left\|x\left(t-\tau_{i L}\right)\right\|^{2} \\
& -\sum_{i=1}^{m} \kappa_{2_{2 i}} e^{-2 \alpha \tau_{i H}}\left\|x\left(t-\tau_{i H}\right)\right\|^{2} \\
& -\sum_{i=1}^{m} \kappa_{2_{4 i}} e^{-2 \alpha v \tau_{i H}}(1-v \mu)\left\|x\left(t-v \tau_{i}(t)\right)\right\|^{2}
\end{aligned}
$$

with $\xi(t)=((p+\beta) / \eta) \delta_{2}(t)$ and $\widetilde{r}(t)=\left((p+\beta)^{2} / \eta\right) \delta_{2}^{2}(t)$.

Taking condition (44) into account, we have

$$
V_{0}\left(t, x_{t}\right) \leq \widetilde{r}(t) .
$$

Therefore, from (33) and (46),

$$
\dot{V}\left(t, x_{t}\right) \leq-2 \alpha V\left(t, x_{t}\right)+\widetilde{r}(t), \quad \forall t \geq 0
$$

which gives

$$
V\left(t, x_{t}\right) \leq V\left(0, x_{0}\right) e^{-2 \alpha t}+\int_{0}^{t} e^{2 \alpha(s-t)} \widetilde{r}(s) d s, \quad \forall t \geq 0 .
$$

Now, if $\delta_{2}(t)$ is bounded for all $t \geq 0$, then there exists $M>0$ such that $\left\|\delta_{2}(t)\right\| \leq M, \forall t \geq 0$. Therefore $\widetilde{r}(t)$ will be bounded, so there exists $M_{1}>0$ such that $\tilde{r}(t) \leq M_{1}, \forall t \geq 0$, with $M_{1}=(p+\beta)^{2} M^{2} / \eta$. So,

$$
V\left(t, x_{t}\right) \leq V\left(0, x_{0}\right) e^{-2 \alpha t}+\frac{M_{1}}{2 \alpha}
$$

and hence, using the fact that

$$
\begin{gathered}
\beta\|x(t)\|^{2} \leq V\left(t, x_{t}\right), \quad t \in \mathfrak{R}^{+}, \\
\|x(t, \phi)\| \leq \sqrt{\frac{V\left(0, x_{0}\right)}{\beta}} e^{-\alpha t}+r_{1}, \quad \forall t \geq 0,
\end{gathered}
$$

with $r_{1}=\sqrt{M_{1} / 2 \alpha \beta}$. This implies that the solution converges to the ball $B\left(0, r_{1}\right)$. 
Next, if $\delta_{2}$ satisfies

$$
\int_{0}^{+\infty} \delta_{2}^{4}(s) d s<+\infty,
$$

then $\widetilde{I}:=\int_{0}^{+\infty} \widetilde{r}^{2}(s) d s<+\infty$.

It follows that

$$
V\left(t, x_{t}\right) \leq V\left(0, x_{0}\right) e^{-2 \alpha t}+\frac{\widetilde{I}^{1 / 2}}{2 \sqrt{\alpha}}, \quad \forall t \geq 0 .
$$

Hence, using (50), one gets

$$
\|x(t, \phi)\| \leq \sqrt{\frac{V\left(0, x_{0}\right)}{\beta}} e^{-\alpha t}+r_{2}, \quad \forall t \geq 0,
$$

with $r_{2}=\sqrt{\widetilde{I}^{1 / 2} / 2 \beta \sqrt{\alpha}}$.

In this case, the solution converges to the ball $B\left(0, r_{2}\right)$.

Remark that, from (45), if we suppose that $\delta_{2}(t)$ tends to zero when $t$ goes to infinity, then $\widetilde{r}(t) \rightarrow 0$ as $t \rightarrow$ $+\infty$; hence the solution of (7) will converge uniformly exponentially to zero when $t$ tends to infinity. Also, note that we can estimate $V\left(0, x_{0}\right)$ as follows.

(1) Estimate $V_{1}\left(0, x_{0}\right)$ as

$$
V_{1}\left(0, x_{0}\right) \leq(p+\beta)\|\phi\|^{2}
$$

(2) Estimate $V_{2}\left(0, x_{0}\right)$ as

$$
\begin{aligned}
V_{2}\left(0, x_{0}\right)= & \sum_{i=1}^{m} \kappa_{2_{1 i}} \int_{-\tau_{i L}}^{0} e^{2 \alpha s}\|x(s)\|^{2} d s \\
& +\sum_{i=1}^{m} \kappa_{2_{2 i}} \int_{-\tau_{i H}}^{0} e^{2 \alpha s}\|x(s)\|^{2} d s \\
& +\sum_{i=1}^{m} \kappa_{2_{3 i}} \int_{-\tau_{i}(t)}^{0} e^{2 \alpha s}\|x(s)\|^{2} d s \\
& +\sum_{i=1}^{m} \kappa_{2_{4 i}} \int_{-v \tau_{i}(t)}^{0} e^{2 \alpha s}\|x(s)\|^{2} d s .
\end{aligned}
$$

(i) Considering $\int_{t-\tau_{i L}}^{t} e^{2 \alpha(s-t)}\|x(s)\|^{2} d s \geq 0$,

$$
\begin{aligned}
& \int_{-\tau_{i L}}^{0} e^{2 \alpha s}\|x(s)\|^{2} d s \\
& \quad \leq\|\phi\|^{2} \int_{-\tau_{i L}}^{0} e^{2 \alpha s} d s=\frac{1}{2 \alpha}\left(1-e^{-2 \alpha \tau_{i L}}\right)\|\phi\|^{2} \\
& \quad \leq \tau_{i L}\|\phi\|^{2} .
\end{aligned}
$$

(ii) Considering $\int_{t-\tau_{i H}}^{t} e^{2 \alpha(s-t)}\|x(s)\|^{2} d s \geq 0$,

$$
\begin{aligned}
& \int_{-\tau_{i H}}^{0} e^{2 \alpha s}\|x(s)\|^{2} d s \\
& \quad \leq\|\phi\|^{2} \int_{-\tau_{i H}}^{0} e^{2 \alpha s} d s=\frac{1}{2 \alpha}\left(1-e^{-2 \alpha \tau_{i H}}\right)\|\phi\|^{2} \\
& \quad \leq \tau_{i H}\|\phi\|^{2} .
\end{aligned}
$$

(iii) Considering $\int_{t-\tau_{i}(t)}^{t} e^{2 \alpha(s-t)}\|x(s)\|^{2} d s \geq 0$,

$$
\begin{aligned}
& \int_{-\tau_{i}(t)}^{0} e^{2 \alpha s}\|x(s)\|^{2} d s \\
& \quad \leq\|\phi\|^{2} \int_{-\tau_{i}(t)}^{0} e^{2 \alpha s} d s=\frac{1}{2 \alpha}\left(1-e^{-2 \alpha \tau_{i}(t)}\right)\|\phi\|^{2} \\
& \quad \leq \tau_{i H}\|\phi\|^{2} .
\end{aligned}
$$

(iv) Considering $\int_{t-\nu \tau_{i}(t)}^{t} e^{2 \alpha(s-t)}\|x(s)\|^{2} d s \geq 0$,

$$
\begin{aligned}
& \int_{-v \tau_{i}(t)}^{0} e^{2 \alpha s}\|x(s)\|^{2} d s \\
& \quad \leq\|\phi\|^{2} \int_{-v \tau_{i}(t)}^{0} e^{2 \alpha s} d s=\frac{1}{2 \alpha}\left(1-e^{-2 \alpha v \tau_{i}(t)}\right)\|\phi\|^{2} \\
& \quad \leq v \tau_{i H}\|\phi\|^{2} \\
& V_{2}\left(0, x_{0}\right) \leq \sum_{i=1}^{m}\left(\kappa_{2_{1 i}} \tau_{i L}+\left(\kappa_{2_{2 i}}+\kappa_{2_{3 i}}+\kappa_{2_{4 i}} \nu\right) \tau_{i H}\right)\|\phi\|^{2} .
\end{aligned}
$$

(3) Estimate $V_{3}\left(0, x_{0}\right)$ as

$$
\begin{aligned}
V_{3}\left(0, x_{0}\right) & \\
= & \sum_{i=1}^{m} \kappa_{3_{1 i}} \tau_{i H} \int_{-\tau_{i H}}^{0} \int_{t_{1}-\tau_{i}\left(t_{1}\right)}^{0} e^{2 \alpha\left(s+\tau_{i H}\right)}\|x(s)\|^{2} d s d t_{1} \\
& +\sum_{i=1}^{m} \kappa_{3_{2 i}} \tau_{i H L} \int_{-\tau_{i H}}^{-\tau_{i L}} \int_{t_{1}-\tau_{i}\left(t_{1}\right)}^{0} e^{2 \alpha\left(s+\tau_{i H}\right)}\|x(s)\|^{2} d s d t_{1} .
\end{aligned}
$$

The first member of $V_{3}\left(0, x_{0}\right)$ is

$$
\begin{aligned}
& \tau_{i H} \int_{-\tau_{i H}}^{0} \int_{t_{1}-\tau_{i}\left(t_{1}\right)}^{0} e^{2 \alpha\left(s+\tau_{i H}\right)}\|x(s)\|^{2} d s d t_{1} \\
& \quad \leq\|\phi\|^{2} \tau_{i H} \int_{-\tau_{i H}}^{0} \int_{t_{1}-\tau_{i H}}^{0} e^{2 \alpha\left(s+\tau_{i H}\right)} d s d t_{1} \\
& \quad \leq \frac{\tau_{i H}}{2 \alpha}\left(\tau_{i H} e^{2 \alpha \tau_{i H}}-\frac{1}{2 \alpha}\left(1-e^{-2 \alpha \tau_{i H}}\right)\right)\|\phi\|^{2} \\
& \quad \leq c_{i H} \tau_{i H}^{3}\|\phi\|^{2} .
\end{aligned}
$$

with $\left(\left(x e^{x}+e^{-x}-1\right) / x^{2} \geq 1.5\right) c_{i H}=\left(\left(2 \alpha \tau_{i H} e^{2 \alpha \tau_{i H}}+\right.\right.$ $\left.\left.e^{-2 \alpha \tau_{i H}}-1\right) /\left(2 \alpha \tau_{i H}\right)^{2}\right)$. 
The second member of $V_{3}\left(0, x_{0}\right)$ is

$$
\begin{aligned}
\tau_{i H L} \int_{-\tau_{i H}}^{-\tau_{i L}} \int_{t_{1}-\tau_{i}\left(t_{1}\right)}^{0} e^{2 \alpha\left(s+\tau_{i H}\right)}\|x(s)\|^{2} d s d t_{1} \\
\quad \leq\|\phi\|^{2} \tau_{i H L} \int_{-\tau_{i H}}^{-\tau_{i L}} \int_{t_{1}-\tau_{i H}}^{0} e^{2 \alpha\left(s+\tau_{i H}\right)} d s d t_{1} \\
\quad \leq \frac{\tau_{i H L}}{2 \alpha}\left(\tau_{i H L} e^{2 \alpha \tau_{i H}}-\frac{1}{2 \alpha}\left[e^{-2 \alpha \tau_{i L}}-e^{-2 \alpha \tau_{i H}}\right]\right)\|\phi\|^{2} \\
\leq \frac{\tau_{i H L}}{(2 \alpha)^{2}}\left(2 \alpha \tau_{i H L} e^{2 \alpha \tau_{i H}}+e^{-2 \alpha \tau_{i H}}-e^{-2 \alpha \tau_{i L}}\right)\|\phi\|^{2} \\
\leq \tau_{i H L}\left(\frac{2 \alpha \tau_{i H} e^{2 \alpha \tau_{i H}}+e^{-2 \alpha \tau_{i H}}-1}{(2 \alpha)^{2}}\right)\|\phi\|^{2} \\
\quad \leq \frac{2 \alpha \tau_{i L} e^{2 \alpha \tau_{i H}}+e^{-2 \alpha \tau_{i L}}-1}{(2 \alpha)^{2}} \\
\leq \tau_{i H L} d_{i H L}\|\phi\|^{2}
\end{aligned}
$$$$
\text { with } d_{i H L}=\left(c_{i H} \tau_{i H}^{2}-c_{i L} \tau_{i L}^{2}\right) \text { and } c_{i L}=\left(2 \alpha \tau_{i L} e^{2 \alpha \tau_{i H}}+\right.
$$$$
\left.e^{-2 \alpha \tau_{i L}}-1\right) /\left(2 \alpha \tau_{i L}\right)^{2} \text {. Consider }
$$$$
V_{3}\left(0, x_{0}\right) \leq \sum_{i=1}^{m}\left(\kappa_{3_{1 i}} c_{i H} \tau_{i H}^{3}+\kappa_{3_{2 i}} \tau_{i H L} d_{i H L}\right)\|\phi\|^{2} \text {. }
$$

Therefore, from (55), (61), and (65), it follows that size

$$
\begin{aligned}
& V\left(0, x_{0}\right) \\
& \leq\left(p+\beta+\sum_{i=1}^{m}\left(\kappa_{2_{1 i}} \tau_{i L}+\left(\kappa_{2_{2 i}}+\kappa_{2_{3 i}}+\kappa_{2_{4 i}} \nu\right) \tau_{i H}\right.\right. \\
& \left.\left.\quad+\kappa_{3_{1 i}} c_{i H} \tau_{i H}^{3}+\kappa_{3_{2 i}} \tau_{i H L} d_{i H L}\right)\right)\|\phi\|^{2}
\end{aligned}
$$

Remark 4. If the delayed nonlinear disturbances are allowed to be of large size in the sense that the constants $\delta_{(\cdot) 2}$ characterizing the upper-bounding functions are large enough in (6), then the radius $r$ of the closed ball $B(0, r)$ becomes accordingly larger according to their values provided in the statement of Theorem 3. That means that if the system is globally exponentially practically stable, then the radius of the residual ball $B(0, r)$ increases as the constants $\delta_{(\cdot) 2}$ increase. As a result, then the uncertainty about how far is the state-trajectory solution from zero becomes larger as those constants increase. Thus, to a larger disturbance, it corresponds to a larger uncertainty about the final deviation of the trajectory from the origin.

Remark 5. On the other hand, if the size of the nonlinear perturbations is allowed to be large in the sense that the constants $\delta_{(\cdot) 1}$ are large enough, then there is trade-off between the values of $\kappa_{1}$ and the maximum matrix measure
$\bar{\mu}(A)$ of $A$ so as to ensure that $\eta>0$ in Theorem 3 . However, note that if the constant $\kappa_{1}$ is large, then the constant $\epsilon$ is requested to be accordingly large. As a result, $A(t)$ should have a sufficiently large absolute stability abscissa for all time in order to compensate for the effects of the perturbations while satisfying the Lyapunov-like matrix equality (9).

Remark 6. Note also from Theorem 3 that the radius of the residual ball $B(0, r)$ also increase with the squared upperbounds of the delays and the squared differences between those upper-bounds and the corresponding delay lowerbounds as well as on certain exponential functions of the maximum delay sizes.

\section{Examples}

Example 1. Consider the nonautonomous system with nonlinear time-delay perturbation (3) with time-varying delay $\tau_{1}(t)=\tau_{1} \cos ^{2}(0.45 t)$ :

$$
\begin{aligned}
f_{1}\left(t, x\left(t-\tau_{1}(t)\right)\right) \\
\quad=\left[\begin{array}{c}
-\delta \sin (t) x_{2}\left(t-\tau_{1}(t)\right)+\delta_{2} * \cos \left(\frac{t}{1+x_{1}^{2}}\right) \\
\delta \cos (t) x_{1}\left(t-\tau_{1}(t)\right)
\end{array}\right],
\end{aligned}
$$

where $\tau_{1}, \delta>0, \delta_{2} \geq 0$ will be chosen later, and

$$
A(t)=\left[\begin{array}{cc}
a(t) & 1 \\
-1 & a(t)
\end{array}\right],
$$

where $a(t)=-0.5 \cos (t)-10 e^{10-\sin (t)}-5.1 e^{-\sin (t)}-1, A_{i}(t)=0$ and $q_{i}=0$ for $(i=1, \ldots, m)$. By computation, we obtain $\bar{\mu}(A) \approx-81033.741$.

$$
\begin{aligned}
& \text { Let } \begin{array}{c}
\beta=0.01, \quad \alpha=1, \quad \mu=0.9, \quad \nu=1.1, \\
\tau_{1 H}=\tau_{1}, \quad \tau_{1 L}=0 \Longrightarrow \tau_{1 H L}=\tau_{1 H}, \\
\delta_{1}=\delta_{11}=\delta, \quad \delta_{12}=\delta_{2}, \\
\kappa_{1}=e^{10}, \quad \kappa_{2_{11}}=0, \quad \kappa_{2_{21}}=0, \quad \kappa_{2_{31}}=e^{10}, \\
\kappa_{2_{41}}=0 \Longrightarrow \kappa_{2_{1}}=e^{10}, \\
\kappa_{3_{11}}=\frac{1}{\tau_{1 H}^{2} \exp \left(2 \alpha \tau_{1 H}\right)}, \quad \kappa_{3_{21}}=0, \\
\Longrightarrow \kappa_{3_{1}}=\frac{1}{\exp \left(2 \alpha \tau_{1 H}\right)} .
\end{array}
\end{aligned}
$$

Then

$$
\begin{aligned}
\epsilon= & 2(p+\beta) \delta_{01}+2 \alpha \beta+\kappa_{2_{1}} \\
& +\kappa_{3_{1}} e^{2 \alpha \tau_{1 H}}+\kappa_{1}=1.02+2 e^{10}>0 .
\end{aligned}
$$


TABLE 1

\begin{tabular}{lccccc}
\hline Delay bound $\left(\tau_{1 H}\right)$ & 2 & 4 & 6 & 8 & 10 \\
\hline Perturbation bound $\delta_{1}$ & 3465.679 & 469.028 & 63.476 & 8.591 & 1.163 \\
\hline Estimated value of $\gamma$ & 2098.897 & 2968.276 & 3635.376 & 4197.767 & 4693.245 \\
\hline
\end{tabular}

We can verify that a solution $P(t)$ is given by

$$
P(t)=\left[\begin{array}{cc}
\frac{e^{\sin (t)}}{10} & 0 \\
0 & \frac{e^{\sin (t)}}{10}
\end{array}\right]
$$

We have $p=\sup _{t \in \mathfrak{R}^{+}}\|P(t)\|=e / 10$,

$$
\begin{gathered}
\delta_{1}<\frac{\sqrt{\kappa_{2_{31}} e^{-2 \alpha \tau_{1 H}}(1-\mu)\left(\kappa_{1}-2 \beta \bar{\mu}(A)\right)}}{(p+\beta)}, \\
\kappa_{1}=e^{10}>2 \beta \bar{\mu}(A)=-1620.67, \\
\eta=\kappa_{1}-2 \beta \bar{\mu}(A)-\frac{(p+\beta)^{2} \delta^{2}}{\kappa_{2_{31}} e^{-2 \alpha \tau_{1 H}}(1-\mu)}>0,
\end{gathered}
$$

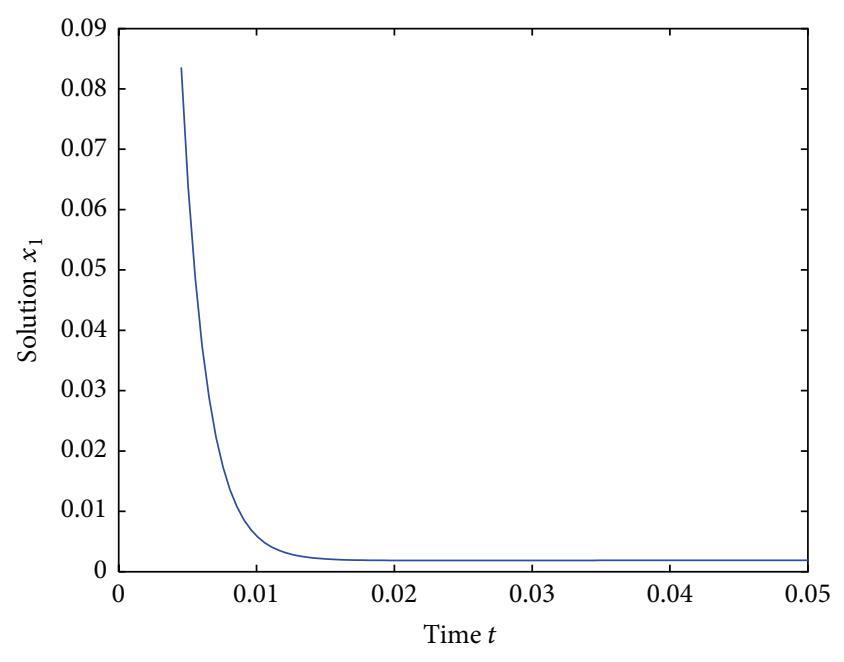

$-x_{1}$

Figure 1: The trajectories of $x_{1}$.

$\eta_{\zeta}=\left(1-\zeta^{2}\right)\left(\kappa_{1}-2 \beta \bar{\mu}(A)\right)>0, \quad$ where $\delta=\zeta \delta_{1}, \quad \zeta<1$,

$$
\begin{gathered}
\gamma=\sqrt{\frac{p+\beta+\kappa_{2_{31}} \tau_{1 H}+\left(c_{1 H} \tau_{1 H} / e^{2 \alpha \tau_{1 H}}\right)}{\beta}} \\
\text { with } c_{1 H}=\frac{2 \alpha \tau_{1 H} e^{2 \alpha \tau_{1 H}}+e^{-2 \alpha \tau_{1 H}}-1}{\left(2 \alpha \tau_{1 H}\right)^{2}} \\
r=\sqrt{\frac{(p+\beta)^{2}}{2 \alpha \beta \eta_{\zeta}} \delta_{2}=\frac{12.95910^{-3}}{\sqrt{1-\zeta^{2}}} \delta_{2} .}
\end{gathered}
$$

If we take $\zeta=0.9$, then $r=29.7310^{-3} \delta_{2}$.

We see that the perturbation bound $\delta_{1}$ in this example is the same as in [5] if $\delta_{2}=0$ and is better than [4], as shown in Table 1. The simulation of this example is shown in Figures 1 and 2 .

Example 2. Consider the following second-order differential system:

$$
\begin{gathered}
\dot{x}_{1}=-5 x_{1}+x_{2}(t-0.1)+\cos \left(\frac{x_{1}}{1+t^{2}}\right) \\
+0.02 \sin \left(x_{1}(t-0.1)+0.5\right), \\
\dot{x}_{2}=-x_{1}(t-0.1)-5 x_{2},
\end{gathered}
$$

with

$$
\begin{gathered}
A=\left[\begin{array}{cc}
-5 & 0 \\
0 & -5
\end{array}\right] ; \quad \lambda_{\max }(A)=-5 ; \quad \bar{\mu}(A)=-5 ; \\
A_{1}=\left[\begin{array}{cc}
0 & 1 \\
-1 & 0
\end{array}\right] ; \quad \tau_{1 L}=0 ; \quad \tau_{1}=\tau_{1 H}=0.1 ; \quad \mu=0 ; \\
f_{0}(t, x)=\left[\begin{array}{c}
\left.\cos \left(\frac{x_{1}}{1+t^{2}}\right)\right] ; \quad \delta_{01}=0 ; \quad \delta_{02}=1 \\
0
\end{array}\right] \quad \begin{array}{c}
0.02 \sin \left(x_{1}(t-0.1)+0.5\right) \\
f_{1}(t, x(t-1))=\left[\begin{array}{c}
0 \\
\delta_{11}=0.02 ; \quad
\end{array} \quad 0.1,\right.
\end{array}
\end{gathered}
$$

$x=\left(x_{1}, x_{2}\right)^{T} \in \mathfrak{R}^{2}$. Hence, using (25), we obtain $\delta_{1}=$ $0.02 ; \delta_{2}=1.1$.

The matrix

$$
P(t)=\left[\begin{array}{ll}
p_{11} & p_{12} \\
p_{12} & p_{22}
\end{array}\right]
$$

can be computed as follows:

$$
\begin{gathered}
p_{11}=p_{22}=-\beta+q_{1}(5-\alpha), \\
p_{12}=\sqrt{q_{1}} \cdot \sqrt{-10 \beta+2 \alpha \beta+q_{1}(\alpha-5)^{2}-\epsilon}
\end{gathered}
$$




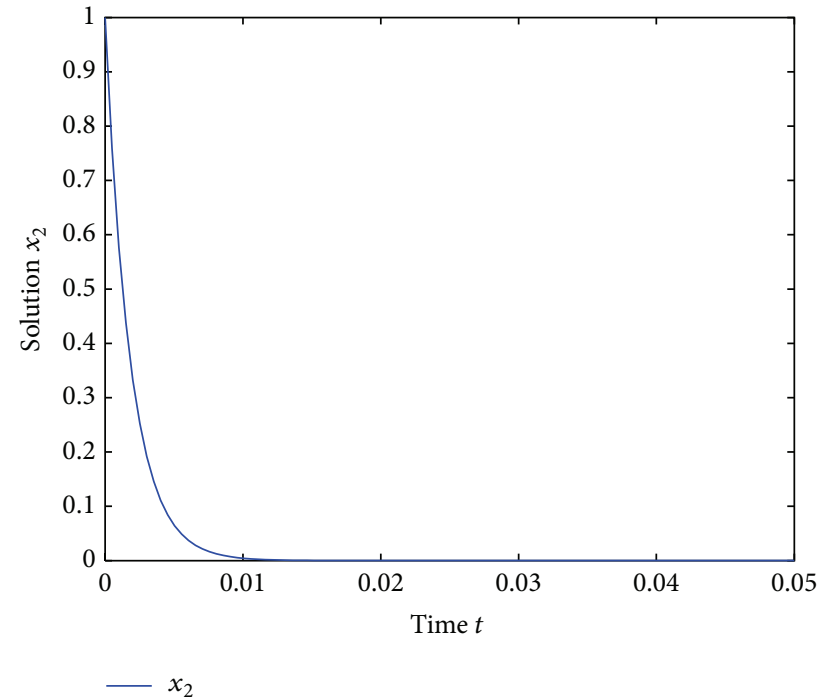

FIgURE 2: The trajectories of $x_{2}$.

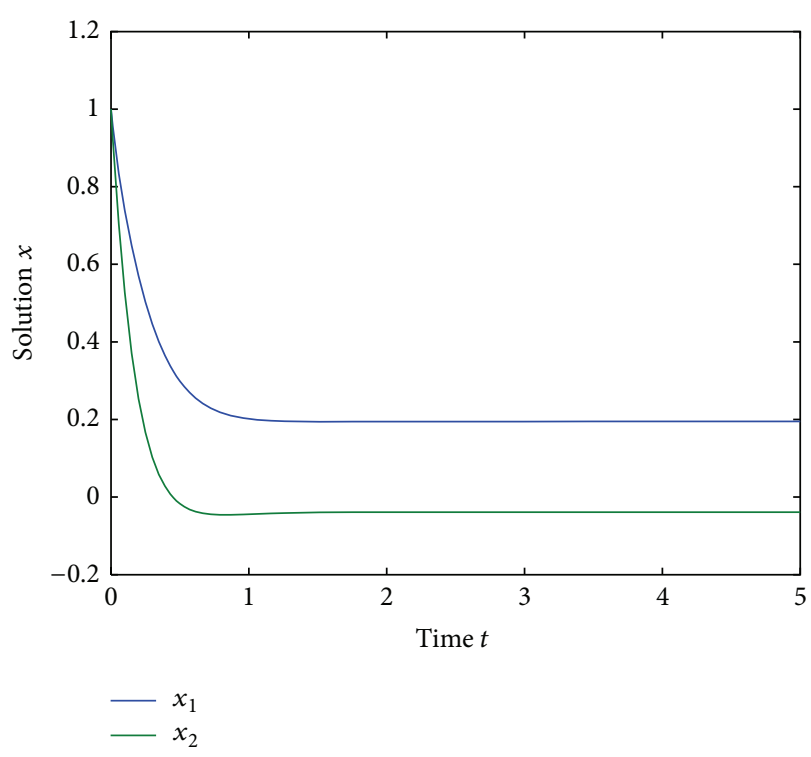

FIGURE 3: Convergence of solutions.

such that the constant

$$
\begin{aligned}
& \left.\alpha<5, \quad q_{1} \in\right] \frac{2 \beta}{(5-\alpha)}, \kappa_{2_{31}} e^{-2 \alpha \tau_{1 H}}[, \\
& 0<\epsilon \leq-10 \beta+2 \alpha \beta+q_{1}(\alpha-5)^{2}, \\
& \alpha=1, \quad \beta=0.001, \quad q_{1}=0.0010489, \\
& \kappa_{1}=0.02>-10 \beta=-0.01, \\
& \kappa_{2_{11}}=0, \quad \kappa_{2_{21}}=0 \text {, } \\
& \kappa_{2_{41}}=0 \Longrightarrow \kappa_{2_{1}}=\kappa_{2_{31}}=0.001282, \\
& \kappa_{3_{11}}=0.16374, \quad \kappa_{3_{21}}=0, \quad \Longrightarrow \kappa_{3_{1}}=0.0016374 \text {. } \\
& p_{11}=p_{22}=0.00319 \text {, } \\
& p_{12}=0.00125 \Longrightarrow p=0.00445 \text {, } \\
& \delta_{1}=0.02<0.0206, \\
& \eta=6.8310^{-4}>0 \text {, } \\
& \gamma=\sqrt{\frac{p+\beta+\kappa_{2_{31}} \tau_{1 H}+\kappa_{3_{11}} c_{1 H} \tau_{1 H}^{3}}{\beta}}=2.416 \\
& \text { with } c_{1 H}=\frac{2 \alpha \tau_{1 H} e^{2 \alpha \tau_{1 H}}+e^{-2 \alpha \tau_{1 H}}-1}{\left(2 \alpha \tau_{1 H}\right)^{2}} \text {, } \\
& r=\frac{(p+\beta) M}{\sqrt{2 \alpha \beta \eta}}=5.13 \quad \text { with } M=\delta_{2}
\end{aligned}
$$

in such a way that condition (12) in Theorem 3 is satisfied.

The result of the simulation of this example is depicted in Figure 3. The evolution of states $x_{1}$ and $x_{2}$ is given. It is shown in Figure 1 that the time-delay perturbed system is

globally uniformly practically exponentially stable toward a neighborhood of the origin.

\section{Conclusion}

Based on improved Lyapunov-Krasovskii functional for perturbed systems with time-varying delay, we have presented new sufficient conditions for global uniformly exponential practical stability toward a certain ball neighborhood of the origin. The perturbations are assumed to be nonlinear, in general, with delayed contributions. The delayed contributions of such perturbations are not necessarily bounded while they are upper-bounded by known nonnegative integrable functions which are linear functions of the various timedelayed state norms. The point delays are assumed to be unknown bounded time-differentiable functions of time with known lower- and upper-bounds and known upper-bounds of their time-derivatives.

\section{Conflict of Interests}

The authors declare that there is no conflict of interests regarding the publication of this paper.

\section{Acknowledgments}

The authors wish to thank the editor and the anonymous reviewers for their valuable and careful comments. The third author is grateful to the Spanish Ministry of Education for its partial support of this work through Grant DPI DPI2012-30651 and is also grateful to the Basque Government for its support through Grants IT378-1 and SAIOTEK SPE13UN039. 


\section{References}

[1] K. Zhou and P. P. Khargonekar, "Stability robustness bounds for linear state-space models with structured uncertainty," IEEE Transactions on Automatic Control, vol. 32, no. 7, pp. 621-623, 1987.

[2] E. Cheres, Z. J. Palmor, and S. Gutman, "Quantitative measures of robustness for systems including delayed perturbations," IEEE Transactions on Automatic Control, vol. 34, no. 11, pp. 1203-1204, 1989.

[3] H. Trinh and M. Aldeen, "On the stability of linear systems with delayed perturbations," IEEE Transactions on Automatic Control, vol. 39, no. 9, pp. 1948-1951, 1994.

[4] H. Trinh and M. Aldeen, "On robustness and stabilization of linear systems with delayed nonlinear perturbations," IEEE Transactions on Automatic Control, vol. 42, no. 7, pp. 1005-1007, 1997.

[5] P. Niamsup, K. Mukdasai, and V. N. Phat, "Improved exponential stability for time-varying systems with nonlinear delayed perturbations," Applied Mathematics and Computation, vol. 204, no. 1, pp. 490-495, 2008.

[6] M. Hammami and I. Ellouze, "A robust detector for a class of uncertain systems," Nonlinear Dynamics and Systems Theory, vol. 8, no. 4, pp. 349-358, 2008.

[7] M. de la Sen, "On the stability of a certain class of linear timevarying systems," The American Journal of Applied Sciences, vol. 2, no. 8, pp. 1240-1245, 2005.

[8] M. de la Sen, "Robust stability of a class of linear timevarying systems," IMA Journal of Mathematical Control and Information, vol. 19, no. 4, pp. 399-418, 2002.

[9] V. N. Phat, "Global stabilization for linear continuous timevarying systems," Applied Mathematics and Computation, vol. 175, no. 2, pp. 1730-1743, 2006.

[10] A. Zevin and M. Pinsky, "Exponential stability and solution bounds for systems with bounded nonlinearities," IEEE Transactions on Automatic Control, vol. 48, no. 10, pp. 1799-1804, 2003.

[11] X.-L. Zhu and G.-H. Yang, "Delay-dependent stability criteria for systems with differentiable time delays," Acta Automatica Sinica, vol. 34, no. 7, pp. 765-771, 2008.

[12] M. de la Sen and N. S. Luo, "On the uniform exponential stability of a wide class of linear time-delay systems," Journal of Mathematical Analysis and Applications, vol. 289, no. 2, pp. 456-476, 2004.

[13] H. R. Karimi, M. Zapateiro, and N. Luo, "New delay-dependent stability criteria for uncertain neutral systems with mixed timevarying delays and nonlinear perturbations," Mathematical Problems in Engineering, vol. 2009, Article ID 759248, 22 pages, 2009.

[14] J.-Z. Zhang, Z. Jin, Q.-X. Liu, and Z.-Y. Zhang, "Analysis of a delayed SIR model with nonlinear incidence rate," Discrete Dynamics in Nature and Society, vol. 2008, Article ID 636153, 16 pages, 2008.

[15] C. Wei and L. Chen, "A delayed epidemic model with pulse vaccination," Discrete Dynamics in Nature and Society, vol. 2008, Article ID 746951, 12 pages, 2008.

[16] Y.-H. Fan and L.-L. Wang, "Permanence for a discrete model with feedback control and delay," Discrete Dynamics in Nature and Society, vol. 2008, Article ID 945109, 8 pages, 2008.

[17] Q. Liu, "Almost periodic solution of a diffusive mixed system with time delay and type III functional response," Discrete
Dynamics in Nature and Society, vol. 2008, Article ID 706154, 13 pages, 2008.

[18] V. N. Phat and P. T. Nam, "Exponential stability criteria of linear non-autonomous systems with multiple delays," Electronic Journal of Differential Equations, vol. 2005, no. 58, pp. 1-8, 2005.

[19] M. de la Sen and A. Ibeas, "On the global asymptotic stability of switched linear time-varying systems with constant point delays," Discrete Dynamics in Nature and Society, vol. 2008, Article ID 231710, 31 pages, 2008.

[20] Y. Chen, W. Bi, and Y. Wu, "Delay-dependent exponential stability for discrete-time BAM neural networks with timevarying delays," Discrete Dynamics in Nature and Society, vol. 2008, Article ID 421614, 14 pages, 2008.

[21] J. H. Park and O. Kwon, "Matrix inequality approach to a novel stability criterion for time-delay systems with nonlinear uncertainties," Journal of Optimization Theory and Applications, vol. 126, no. 3, pp. 643-656, 2005.

[22] V. N. Phat and P. Niamsup, "Stability analysis for a class of functional differential equations and applications," Nonlinear Analysis: Theory, Methodse Applications, vol. 71, no. 12, pp. 6265-6275, 2009.

[23] A. Benabdallah, I. Ellouze, and M. A. Hammami, "Practical stability of nonlinear time-varying cascade systems," Journal of Dynamical and Control Systems, vol. 15, no. 1, pp. 45-62, 2009.

[24] V. Lakshmikantham, S. Leela, and A. A. Martynyuk, Practical Stability of Nonlinear Systems, World Scientific, 1990.

[25] V. Lakshmikantham and Y. Zhang, "Strict practical stability of delay differential equation," Applied Mathematics and Computation, vol. 118, no. 2-3, pp. 275-285, 2001.

[26] M. Corless, "Guaranteed rates of exponential convergence for uncertain systems," Journal of Optimization Theory and Applications, vol. 64, no. 3, pp. 481-494, 1990.

[27] M. Corless and G. Leitmann, "Bounded controllers for robust exponential convergence," Journal of Optimization Theory and Applications, vol. 76, no. 1, pp. 1-12, 1993. 


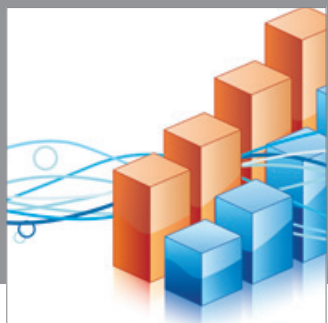

Advances in

Operations Research

mansans

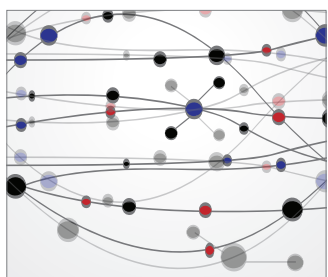

The Scientific World Journal
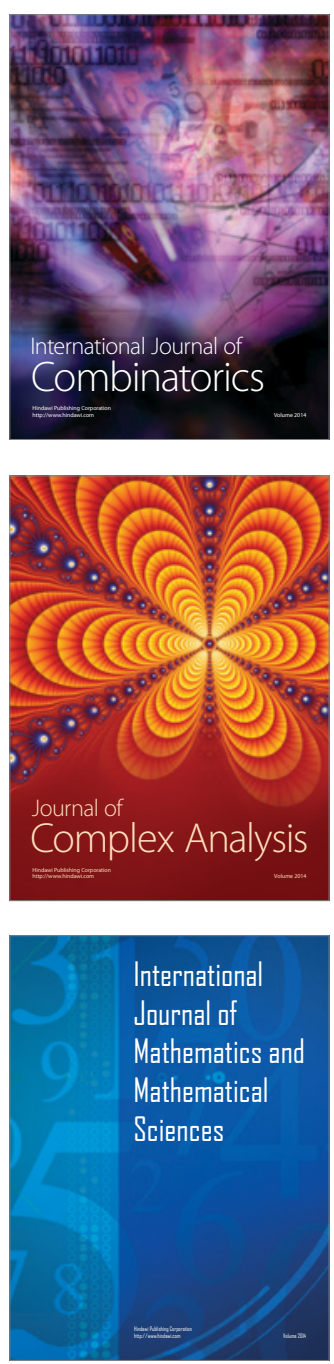
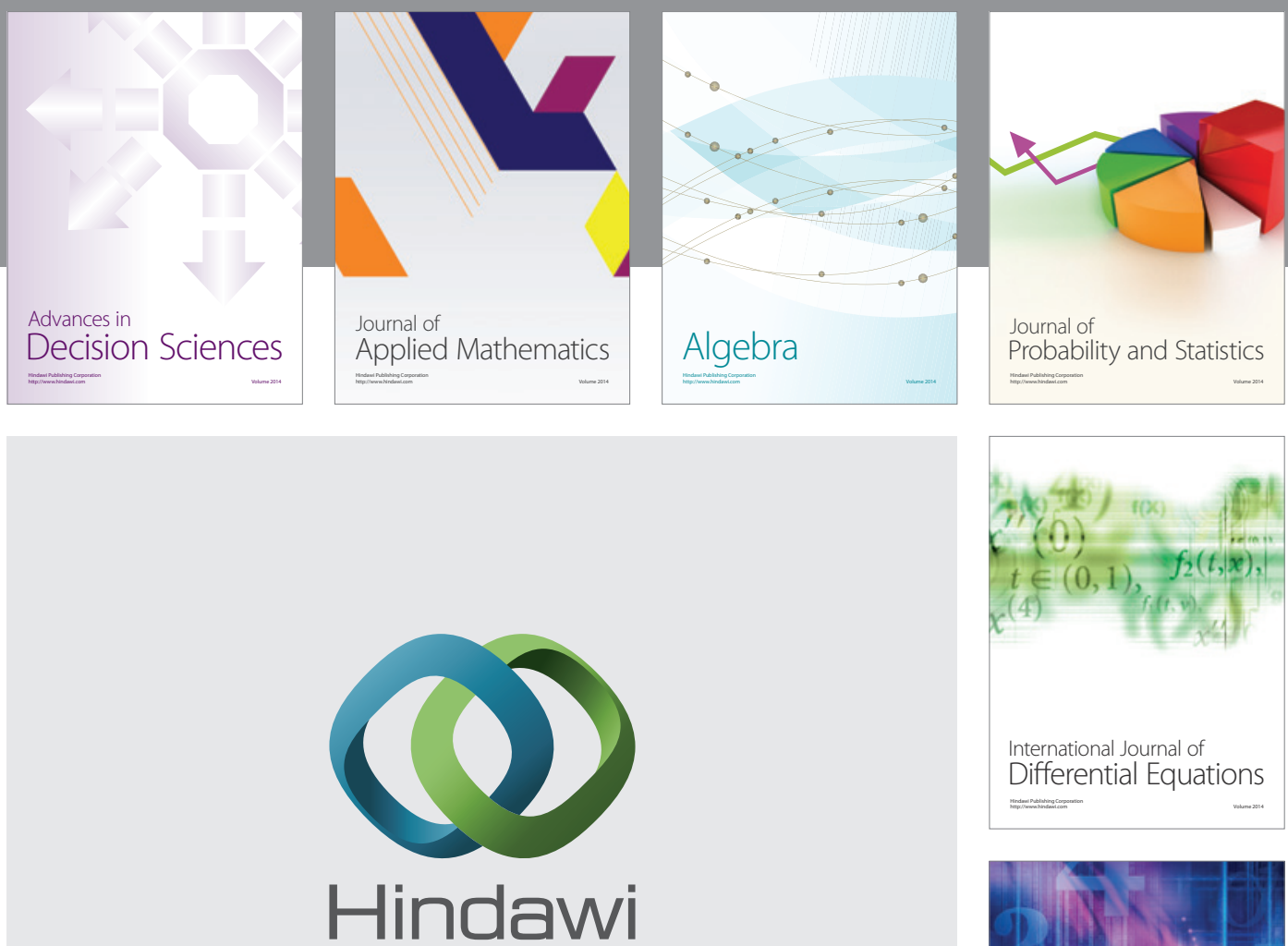

Submit your manuscripts at http://www.hindawi.com
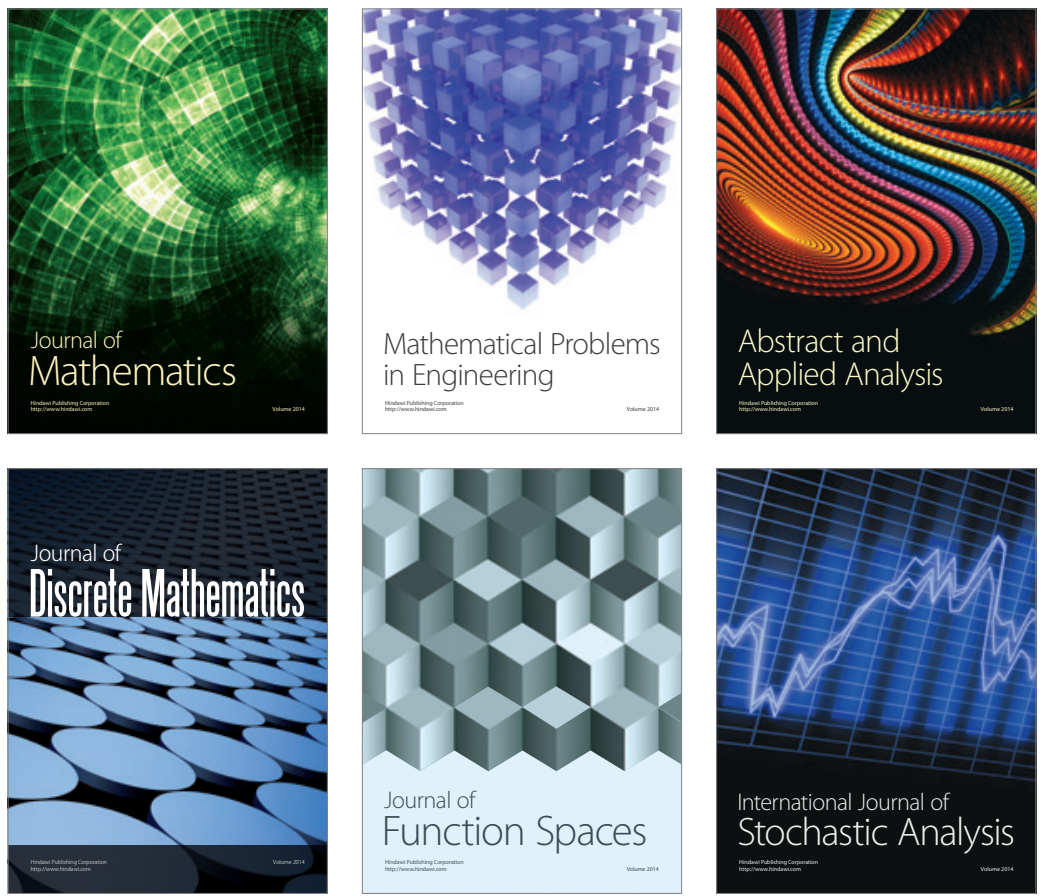

Journal of

Function Spaces

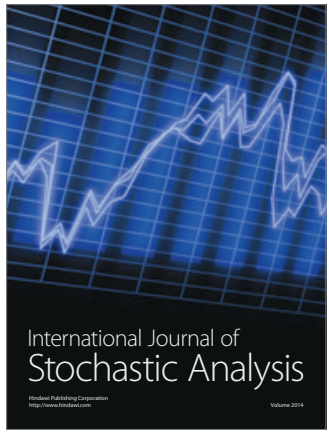

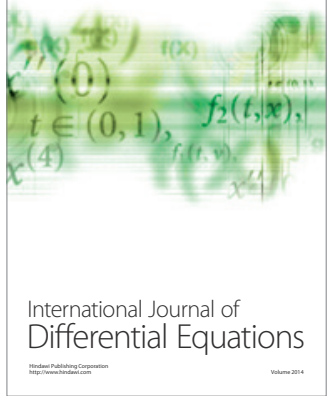
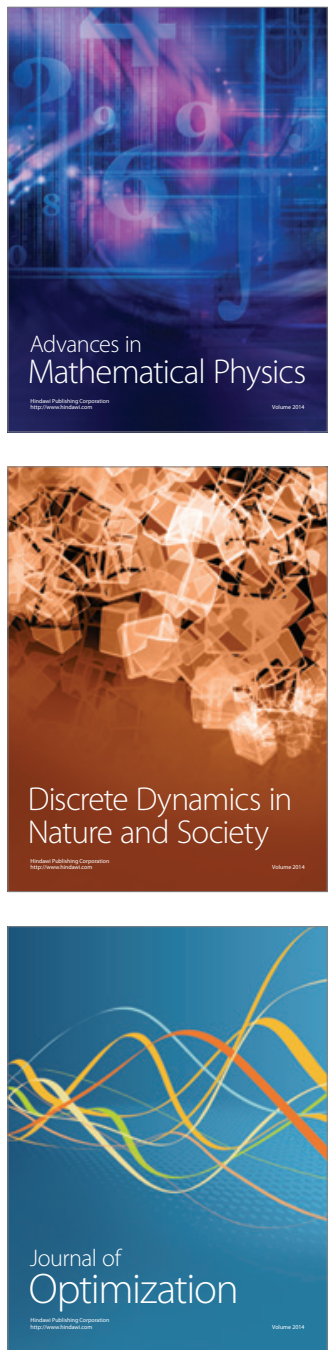